\title{
Estime de soi, sentiment d'autoefficacité et intention de décrocher au collégial
}

Stéphane Thibodeau, Elisabeth Giguère, Sonia Lefebvre and Eric Frenette

\section{(2) OpenEdition}

1 Journals

Electronic version

URL: https://journals.openedition.org/ripes/3359

DOI: $10.4000 /$ ripes.3359

ISSN: 2076-8427

Publisher

Association internationale de pédagogie universitaire

Electronic reference

Stéphane Thibodeau, Elisabeth Giguère, Sonia Lefebvre and Eric Frenette, "Estime de soi, sentiment d'autoefficacité et intention de décrocher au collégial", Revue internationale de pédagogie de

l'enseignement supérieur [Online], 37(3) | 2021, Online since 08 November 2021, connection on 27

September 2022. URL: http://journals.openedition.org/ripes/3359 ; DOI: https://doi.org/10.4000/ripes. 3359

This text was automatically generated on 27 September 2022

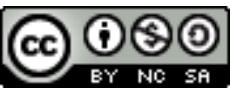

Creative Commons - Attribution-NonCommercial-ShareAlike 4.0 International - CC BY-NC-SA 4.0 https://creativecommons.org/licenses/by-nc-sa/4.0/ 


\title{
Estime de soi, sentiment d'autoefficacité et intention de décrocher au collégial
}

\author{
Stéphane Thibodeau, Elisabeth Giguère, Sonia Lefebvre and Eric Frenette
}

\section{Introduction}

1 Même si les différents acteurs du système d'éducation travaillent sans cesse à son amélioration, la population québécoise demeure insatisfaite par rapport aux taux de décrochage ou de réussite décevants des jeunes. Pour trouver des solutions à ces problèmes, les chercheurs se penchent, notamment, sur le système, les enseignants ou les élèves. La présente étude s'inscrit dans cette dernière perspective en s'intéressant à la condition psychologique des étudiants du collégial.

2 Plus précisément, la présente recherche est exploratoire et vise à mettre de l'avant les relations entre l'estime de soi et l'intention de décrocher des collégiens, de même que d'identifier le rôle que peut jouer le sentiment d'autoefficacité scolaire de ces derniers dans cette relation. Les résultats de cette recherche permettront aux divers intervenants du niveau collégial de mieux intervenir auprès des étudiants côtoyant la perspective du décrochage.

\section{Problématique}

\subsection{Décrochage scolaire au collégial}

3 Le taux de diplomation serait en baisse dans les collèges de la province de Québec (Michel Perron, cité dans Dion-Viens, 2017). En fait, les taux de décrochage scolaire au niveau collégial sont alarmants (Rapport à l'école au collégial, 2021). Selon Shaienks et al. (2008), le décrochage au collégial est le fait, pour un étudiant, d'avoir entrepris des études dans une institution d'enseignement supérieur, mais qui ne les poursuit plus 
et n'a jamais obtenu de diplôme de cet établissement. Pour l'année scolaire 2008-2009, bien que $64 \%$ des jeunes entraient au collège, 37,5\% d'entre eux quittaient leurs études avant l'obtention d'un diplôme (Ministère de l'Éducation du Loisir et du Sport [MELS], 2010). De plus, une étude menée par Roy, Bouchard et Turcotte (2010) permet de constater qu'un étudiant du collégial sur sept songerait à abandonner ses études. Finalement, plusieurs constats sont ressortis d'une enquête visant à favoriser la réussite éducative et l'insertion socioprofessionnelle menée dans les régions de Lanaudière, de la Mauricie et du Saguenay-Lac-Saint-Jean en 2014 (Gaudreault et al., 2014). En effet, selon cette enquête, plus de $40 \%$ des cégépiens échouaient au moins un cours au premier trimestre. Plus du tiers $(36 \%)$ penseraient à décrocher lors de sa première session. Finalement, $25 \%$ des apprenants qui étaient inscrits dans une formation préuniversitaire n'ont pas terminé leur programme cinq ans plus tard. En ce qui a trait au secteur technique, la statistique grimpe à $38 \%$.

\subsection{Effets du décrochage scolaire ${ }^{1}$}

4 Que ce soit pour l'individu ou la société, le décrochage scolaire semble amener de lourdes conséquences. Tout d'abord, selon le Comité Mauricien sur la Persévérance et la Réussite Scolaire (COMPERES, 2013), il affecterait considérablement le jeune dans son individualité. En effet, on constate un état de santé général moins bon et un risque de dépression à l'âge adulte plus élevé chez les non-diplômés que chez les diplômés (COMPERES, 2013). Le décrochage scolaire aurait également un effet sur la motivation et l'engagement des individus (COMPERES, 2013). Ne détenant pas de diplôme, ces derniers seraient conscients que leurs chances de se tailler une place de choix sur le marché du travail sont réduites. Ayant des lacunes en ce qui a trait au savoir et au savoir-faire, les décrocheurs pourraient donc éprouver un manque de confiance en l'avenir. Aussi, le décrochage scolaire augmenterait les risques de marginalité et d'exclusion sociale (Comité Régional pour la Valorisation de l'Éducation, CRÉVALE, 2013). En effet, les décrocheurs semblent moins participer à la vie d'une société, c'est-àdire qu'ils seraient moins engagés, que ce soit sur les plans sociaux ou politiques. En ce qui concerne l'exclusion, celle-ci peut prendre différentes formes. La délinquance, la criminalité, la toxicomanie, la prostitution, l'itinérance et les problèmes psychosociaux en sont de bons exemples. Finalement, les jeunes qui quittent précocement le milieu scolaire courent 1,7 fois plus de risque de faire partie de la population carcérale (CRÉVALE, 2013).

5 Le décrochage scolaire peut engendrer des conséquences néfastes pour l'individu, mais tout autant pour la société. En effet, les personnes qui quittent l'école hâtivement hypothèqueraient considérablement le développement d'une région (CRÉVALE, 2013). Par leur manque de qualifications, ils rendent plus difficile le recrutement d'une maind'œuvre compétente pour les entreprises. De plus, ce problème engendre d'énormes coûts financiers pour l'ensemble de la société québécoise. En effet, l'état québécois dépenserait plus de 1,9 milliard de dollars par an pour contrer le décrochage scolaire (Ménard, 2009). Des salaires manquants, des revenus de taxes inexistants, des augmentations des coûts de services de santé, des augmentations des prestations d'assurance-emploi et une hausse des coûts de prévention et de répression du crime ne sont que quelques exemples des nombreuses conséquences qu'amène le décrochage scolaire sur le plan sociétal (COMPERES, 2013). 


\subsection{Causes du décrochage scolaire}

6 Le décrochage scolaire n'est pas une décision momentanée, mais plutôt « un long processus interactif entre l'élève et l'environnement dans lequel il évolue » (Robertson et Collerette, 2005, p. 688). Il n'y a donc pas de profil et de cause unique qui caractérisent l'étudiant potentiellement à risque de décrocher (Robertson et Collerette, 2005). La recension d'écrits scientifiques de Fortin et al. (2004) permet de faire ressortir trois types de facteurs associés au décrochage scolaire. Il s'agit des facteurs institutionnels, interpersonnels et personnels.

7 Au plan institutionnel, l'organisation des lieux, le climat, les différents programmes offerts sont de bons exemples. Ensuite, au plan interpersonnel, Fortin et al. (2004) relèvent que des facteurs familiaux, notamment un style de vie non conventionnel (drogues ou abus d'alcool à la maison), le peu d'attentes face à la réussite scolaire de la part des parents, un manque de supervision et d'encouragement ainsi qu'un manque de chaleur dans les rapports parents-enfants sont fortement associés au décrochage scolaire. De plus, la qualité de la relation enseignant-élève, de par les comportements qui peuvent en découler, peut nuire à la réussite et à la persévérance scolaire de l'apprenant. Finalement, le rejet par les pairs, l'isolement social et la fréquentation de pairs ayant peu d'aspirations scolaires sont d'autres facteurs interpersonnels reliés au décrochage. Enfin, au plan personnel, les difficultés d'apprentissage, l'échec et le retard scolaire, le manque de motivation, les troubles de comportement ainsi que de faibles habiletés intellectuelles et verbales semblent amener un étudiant à quitter le milieu scolaire (Fortin et al., 2004).

Le décrochage scolaire semble donc être un phénomène complexe qui peut s'expliquer par plusieurs facteurs ou par une combinaison de ces derniers. Par conséquent, la présente recherche vise à explorer les facteurs personnels des étudiants au collégial qui peuvent les amener à décrocher. Parmi ces facteurs, deux semblent particulièrement intéressants et largement étudiés auprès d'autres apprenants. Il s'agit de l'estime de soi et du sentiment d'autoefficacité.

9 Pour ce qui est de l'estime de soi, les écrits scientifiques sur le monde scolaire montrent, entre autres, que l'estime de soi est reliée à la motivation des apprenants (Gurtner, Gorga, Monnard et Ntamakiliro, 2001; Shi et Steen, 2012). Muchielli (2006) considère même qu'elle est la composante principale de la motivation. Quant au sentiment d'autoefficacité, il a été étudié dans de nombreux domaines, notamment dans le scolaire. En effet, le sentiment d'autoefficacité a été examiné en lien avec l'apprentissage de diverses matières scolaires (Bouffard-Bouchard, 1992; Capron, 2012; Joet, 2010). Il a également capté l'intérêt des chercheurs relativement à l'engagement et la performance (Galand et Vanlede, 2004), la persévérance (Janosz et al., 1997), le rendement (Dumont, Leclerc et Deslandes, 2003) et la réussite scolaire des apprenants (Lecompte, 2004).

10 À la lumière des écrits scientifiques, l'estime de soi et le sentiment d'autoefficacité ont été mis en relation avec des variables tels que l'intention de quitter (Alivernini et Lucidi, 2011; Murray et Kennedy-Lightsey, 2013), la mobilisation scolaire (Bardou et al., 2012), la réussite scolaire (Adeyinka et al., 2011; Alivernini et Lucidi, 2011; Hsieh, Sullivan et Guerra, 2007) et l'échec scolaire (Perterson-Graziose et al., 2013). Bien que ces variables soient proches du phénomène à l'étude, il ne semble pas exister, à travers la littérature, de recherche portant sur le lien entre l'estime de soi, le sentiment 
d'autoefficacité et le décrochage scolaire, notamment chez les étudiants du collégial. Partant de ces considérations, on peut se poser la question suivante: quelle est l'influence de l'estime de soi et du sentiment d'autoefficacité sur le décrochage scolaire des étudiants du collégial?

\section{Cadre de référence}

\subsection{Décrochage scolaire et estime de soi}

11 Selon Legendre (2005), le concept de décrochage scolaire renvoie à celui d'abandon scolaire. Il définit l'abandon scolaire au collégial comme étant le fait, pour un étudiant, de quitter définitivement un cours ou un programme d'études. On peut considérer le décrochage scolaire et l'abandon scolaire comme deux expressions qui désignent l'interruption définitive ou temporaire des études avant l'obtention d'une reconnaissance des acquis que ce soit un diplôme, un certificat, une attestation des études, etc. de la part d'une institution d'enseignement (Projet Partenaires pour la Réussite Éducative en Estrie, PRÉE, 2012). Comme il est difficile de repérer les décrocheurs sur le terrain et que l'intention de décrocher serait un fort prédicteur du décrochage scolaire (Hardre et Reeve, 2003), à l'instar d'Alivernini et Lucidi (2011), il semble justifié d'étudier l'intention de décrocher au lieu du décrochage scolaire.

Pour ce qui est de l'estime de soi, Harter (1999) la définit comme un ensemble d'évaluations générales et d'attitudes de l'individu à l'égard de sa propre personne. Cette chercheure propose une perspective multidimensionnelle et dynamique de ce concept. En effet, à l'estime globale, elle ajoute l'estime de soi par rapport à divers domaines. Harter (1999) différencie donc l'estime de soi générale de l'estime de soi reliée à des domaines, comme par exemple, celui des sports. Elle spécifie que l'estime de soi globale n'est pas une «moyenne » ou un «total» des différents domaines, mais plutôt une évaluation globale que la personne se fait d'elle-même.

La théorie de Harter (1999) propose que les différents domaines soient reliés aux différentes périodes de la vie d'un individu. Ces périodes, au nombre de six, sont les suivantes: petite enfance, du milieu à la fin de l'enfance, adolescence, années universitaires, du début au milieu de l'âge adulte et âge adulte avancé. Normalement, lors de leur passage au collégial, l'âge des étudiants se situe entre 16 et 18 ans. La période retenue dans le cadre de la présente étude est donc l'adolescence. Pour cette période, la théorie de Harter (1999) suggère neuf dimensions, soit l'estime de soi générale (p. ex., par rapport à soi, à sa vie) et celle relative à huit domaines : scolaire (p. ex. par rapport à son intelligence, à sa rapidité d'exécution des travaux), athlétique (p. ex. par rapport à sa réussite dans tous les sports, dans de nouveaux sports), travail (p. ex. par rapport à sa réussite dans un emploi, à l'obtention de ce dernier) et ceux reliés à l'apparence physique (p. ex. par rapport à son apparence, à son corps), à l'acceptation sociale (p. ex. par rapport à sa facilité à se faire des amis, au nombre de ces derniers), à la conduite/moralité (p. ex. par rapport à sa façon de faire les choses, à ses comportements), aux amitiés profondes (p. ex. par rapport à sa capacité de se faire des amis, des amis en qui il peut avoir confiance) ainsi qu'aux relations sentimentales (p. ex. par rapport au fait de mériter l'amour, à être en mesure de sortir avec quelqu'un qui lui plait). 

les domaines les plus importants pour lui est un déterminant majeur de son estime de soi globale. Cette estime de soi globale est fortement liée aux affects de l'adolescent, tels que la gaité et les symptômes dépressifs. Il semble donc juste de croire qu'une faible estime de soi chez l'adolescent pourrait l'amener à décrocher. Janosz (2000) appuie cette idée, c'est-à-dire que les affects négatifs chez l'adolescent augmenteraient le risque de décrochage scolaire.

Aussi, certaines recherches indiquent des relations entre l'estime de soi et différentes variables reliées au milieu scolaire. En effet, l'étude menée par Bardou et al. (2012) révèle que l'estime de soi est reliée positivement à la mobilisation scolaire. L'estime de soi serait aussi corrélée négativement à l'échec scolaire. C'est ce que soutiennent les résultats de la recherche menée par Perterson-Graziose et al. (2013). L'étude de Kandemir et al. (2014), quant à elle, supporte l'idée d'une relation négative entre l'estime de soi et la procrastination. Finalement, l'estime de soi des étudiants serait un antécédent indirect de l'intention de quitter le milieu scolaire (Murray et KennedyLightsey, 2013).

\subsection{Décrochage scolaire, estime de soi et sentiment d'autoefficacité}

Bandura (2007) définit le sentiment d'autoefficacité comme étant la croyance de l'individu en sa capacité d'organiser et d'exécuter la ligne de conduite requise pour produire des résultats souhaités. Même si le sentiment d'autoefficacité peut être généralisé à diverses activités, il est généralement étudié en fonction d'une tâche précise (Choi, 2003; Luszczynska, Gutiérrez-Dona et Schwarzer, 2005). Dans la présente recherche, chez des adolescents, on s'intéresse aux domaines scolaire, émotionnel et social comme le suggèrent Suldo et Shaffer (2007). Le sentiment d'autoefficacité scolaire réfère aux croyances de l'individu par rapport à sa compétence scolaire, plus précisément à l'habileté de réussir à l'école ainsi qu'à l'adoption de comportements favorisant l'apprentissage (Suldo et Shaffer, 2007). Pour ce qui est du sentiment d'autoefficacité émotionnelle, ce sont les croyances qui concernent la compétence dans le contrôle des émotions (Suldo et Shaffer, 2007). Autrement dit, les croyances de la personne dans sa capacité, par rapport à ses émotions et celles des autres, à les percevoir, les utiliser, les comprendre et les gérer (Kirk, Schutte et Hine, 2008). En ce qui concerne le sentiment d'autoefficacité sociale, il s'agit des croyances qui concernent la compétence à développer et maintenir des relations sociales (Suldo et Shaffer, 2007). Plus précisément, les croyances relatives au fait d'être capable de répondre avec compétence à diverses situations interpersonnelles (Bandura, 1997), donc de croire en ses capacités sociales (Coleman, 2003).

Selon la théorie de Bandura (2007), le sentiment d'autoefficacité influence façon d'agir, de faire des choix de l'individu. Ce dernier entreprend des activités et sélectionne des environnements qu'il estime être en mesure d'affronter. À l'inverse, il aura tendance à éviter les situations où ses capacités seraient surpassées. Il est donc légitime de croire qu'un étudiant qui possède un faible sentiment d'autoefficacité aura tendance à éviter les échecs scolaires, donc à éventuellement décrocher de l'environnement collégial. Aussi, le sentiment d'autoefficacité a un effet sur l'autorégulation de la motivation qui, elle, permet à l'individu de déterminer ce qu'il est en mesure d'accomplir. Dans ce 
contexte, il serait juste de penser qu'un adolescent qui a un faible sentiment d'autoefficacité serait moins motivé à poursuivre ses études, l'inverse étant aussi vrai.

Les résultats des recherches recensées présentent des relations entre le sentiment d'autoefficacité scolaire et d'autres variables reliées au domaine scolaire. En effet, à la lumière des résultats de leurs études, Adeyinka et al. (2011), Alivernini et Lucidi (2011) ainsi que Hsieh et al. (2007) soutiennent que le sentiment d'autoefficacité scolaire prédit la réussite scolaire. Alivernini et Lucidi (2011) ajoutent que le sentiment d'autoefficacité scolaire annonce l'intention de décrocher. Finalement, l'étude de Kandemir et al. (2014), menée auprès d'étudiants turcs, montre une corrélation entre le sentiment d'autoefficacité scolaire et la procrastination. Les résultats des recherches recensées présentent aussi des relations entre les sentiments d'autoefficacité émotionnelle et sociale et des variables reliées au domaine scolaire. En effet, le sentiment d'autoefficacité émotionnelle est relié à la motivation scolaire (Faurie, 2012) et au succès académique (Qualter et al., 2012) alors que sentiment d'autoefficacité sociale présente des relations avec le succès académique (Ladd, 1990; Qualter et al., 2012).

Les résultats des travaux antérieurs permettent également de mettre en lumière les liens existants entre l'estime de soi et le sentiment d'autoefficacité. En effet, les études recensées (Kandemir et al., 2014; Khan et al., 2015; McKay, Dempster et Byrne, 2014; Peterson-Graziose et al., 2013) font ressortir des relations positives entre ces deux variables. Aussi, les résultats de l'étude McKay et al. (2014) montrent que l'estime de soi prédit le sentiment d'autoefficacité, alors que ceux de la recherche de Hajloo (2014) indique que c'est l'autoefficacité qui prédit l'estime de soi.

Partant de ces considérations, il semble exister des relations entre l'estime de soi et le décrochage scolaire, entre le sentiment d'autoefficacité et le décrochage scolaire et, également, entre l'estime de soi et le sentiment d'autoefficacité. Il semble juste de croire que le sentiment d'autoefficacité puisse jouer un rôle dans les relations entre l'estime de soi et le décrochage scolaire. Comme mentionné ci-dessus, à l'instar d'Alivernini et Lucidi (2011), l'intention de décrocher est étudiée pour mieux comprendre le décrochage scolaire. L'objectif de la présente recherche consiste donc à explorer les relations entre l'estime de soi et l'intention de décrocher d'étudiants du collégial, de même que le rôle de leur sentiment d'autoefficacité dans ces relations.

\section{Méthodologie}

\subsection{Participants}

Tous les étudiants, provenant de trois établissements collégiaux (deux publics et un privé), ont été sollicités. Un courriel les invitant à participer à la recherche a été envoyé par l'intermédiaire de leur collège. Un rappel a été fait deux semaines après la demande initiale. L'échantillon comprend 391 participants ( $25,3 \%$ de garçons et $74,7 \%$ de filles) âgés entre 16 et 25 ans $(M=19,56)$. De cet échantillon, $73,3 \%$ des participants fréquentent le secteur public et $26,7 \%$ le secteur privé. Une majorité d'étudiants sont inscrits au secteur technique (52,8\%), alors que les autres proviennent de programmes préuniversitaires ( $25,5 \%$ des sciences humaines et $21,8 \%$ des sciences natures). En ce qui a trait au nombre de sessions complétées, $35,8 \%$ des participants en ont terminé moins de deux, $35 \%$ entre trois et quatre et, finalement, $29,2 \%$ quatre ou plus. 


\subsection{Instruments} Québec par Bouffard et al. (2002) auprès d'étudiants du secondaire, permet de mesurer l'estime de soi des participants. Il semble judicieux de penser que ce questionnaire puisse être utilisé avec des étudiants du niveau collégial, car ces derniers sont à la fin de l'adolescence. Il permet de mesurer l'estime de soi globale des adolescents et celle relative à aux huit domaines présentés dans le cadre de référence. Le SPPA se compose de 45 items répartis selon neuf dimensions (cinq items par dimensions): scolaire, athlétique, travail, apparence physique, acceptation sociale, conduite/moralité, amitiés profondes, relations sentimentales et estime de soi globale. Chaque item se présente sous la forme de paires d'énoncés contrastant deux types de jeunes. Le participant doit choisir celui qui le représente le mieux et indiquer comment cela le représente (un peu comme moi ou tout à fait comme moi), pour un total de quatre choix de réponse. Les indices de consistance interne de la version traduite par Bouffard et al. (2002) sont: scolaire $(\alpha=0,77)$, athlétique $(\alpha=0,91)$, travail $(\alpha=0,73)$, apparence physique $(\alpha=0,91)$, acceptation sociale $(\alpha=0,83)$, conduite/moralité $(\alpha=0,76)$, amitiés profondes $(\alpha=0,84)$, relations sentimentales $(\alpha=0,63)$ et estime de soi globale $(\alpha=0,85)$. Dans le cadre de la présente étude, les indices de cohérence interne sont considérés adéquats : scolaire ( $\alpha=$ $0,73)$, athlétique $(\alpha=0,87)$, travail $(\alpha=0,71)$, apparence physique $(\alpha=0,90)$, acceptation sociale $(\alpha=0,77)$, conduite/moralité $(\alpha=0,76)$, amitiés profondes $(\alpha=0,80)$, relations sentimentales $(\alpha=0,66)$ et estime de soi globale $(\alpha=0,82)$.

Le Self-Efficacy Questionnaire for Children (SEQ-C) a été élaboré par Muris (2001) pour les enfants, alors que l'adaptation de Suldo et Shaffer (2007) s'adresse aux adolescents. Cette dernière version, traduite en français, s'applique donc aux participants de la présente recherche. Elle est composée de trois dimensions (sept items par dimensions) soit, scolaire, émotionnelle et sociale. Ce questionnaire propose 21 items, évalués à partir d'une échelle en cinq points (1- pas du tout à 5- très bien). Les indices de consistance interne rapportés par Suldo et Shaffer (2007) sont: scolaire $(\alpha=0,82)$, émotionnelle $(\alpha=0,79)$ et sociale $(\alpha=0,73)$. Dans la présente étude, les indices de consistance interne sont considérés adéquats : scolaire $(\alpha=0,81)$, émotionnelle $(\alpha=$ $0,81)$ et sociale $(\alpha=0,73)$.

L'intention de décrocher se mesure à l'aide de la traduction des trois énoncés proposés par Hardre et Reeve (2003) : "Je considère parfois l'option de décrocher de l'école ", "J'ai l'intention de décrocher de l'école» et "Année après année, je suis parfois incertain de continuer mes études ». Cet instrument présente une échelle de type Likert en sept points (1- pas du tout vrai à 7- totalement vrai). Hardre et Reeve (2003) rapportent un indice de consistance interne de 0,79. Dans la présente étude, il est de 0,84 .

\subsection{Analyses statistiques}

Dans un premier temps, la moyenne, l'écart-type, le kurtosis et l'aplatissement pour chacune des échelles des trois concepts à l'étude seront présentés, suivis des corrélations entre ces dernières. Par la suite, la structure factorielle des questionnaires sera vérifiée à l'aide d'analyses par équations structurelles. Enfin, un modèle

Revue internationale de pédagogie de l'enseignement supérieur, 37(3) | 2021 
acheminatoire sera testé à l'aide d'analyses par équations structurelles. Ces analyses seront effectuées à l'aide de la version 6.2 du logiciel EQS (Bentler et Wu, 2006). La méthode d'estimation du maximum de vraisemblance (ML) avec la correction de Lee et al. (1995), l'option pour variables catégorielles et l'option Robust sont utilisées pour les analyses (Byrne, 2006). Cette approche utilisée dans EQS pour traiter des variables catégorielles se distingue des autres logiciels d'équations structurelles. Elle est relativement stable pour de petits échantillons, ce qui n'est pas le cas de la méthode full information (Byrne, 2006). Deux analyses complémentaires permettent, au besoin, d'optimiser le modèle. Le Wald Test (Chou et Bentler, 1990) indique si des liens présents dans le modèle doivent être retirés. Quant au LMTest (Chou et Bentler, 1990), il indique si des liens non définis dans le modèle doivent être ajoutés. Étant donné le nombre limité de répondants pour estimer le modèle acheminatoire et le nombre de paramètres à estimer, il a été décidé de recourir à l'utilisation d'indicateurs pour les variables latentes. Les indicateurs ont été créés suivant la recommandation de Little et al. (2013) d'utiliser la moyenne des items pour former les indicateurs (soit un indicateur par échelle). L'utilisation d'indicateurs pour les variables latentes est une méthode aussi fiable que celle utilisant tous les énoncés pour évaluer l'adéquation de modèle théorique (Marsh et al., 1997).

Trois statistiques d'ajustement sont utilisées pour vérifier l'ajustement du modèle aux données. L'indice d'adéquation non normé (Non Normed Fit Index, NNFI) proposé par Tucker et Lewis (1973), ainsi que l'indice d'adéquation comparatif (Comparative Fit Index, CFI; Bentler, 1990) varient entre 0 et 1 et proviennent de la comparaison entre le modèle nul (modèle n'ayant aucun lien entre les variables). Un modèle présentant une valeur supérieure à 0,90 est jugé comme acceptable (Schumacker et Lomax, 1996), alors qu'un modèle présentant une valeur supérieure à 0,95 est considéré appréciable (Hu et Bentler, 1999, cité dans Tabachnick et Fidell, 2007). Pour le RMSEA (Root Mean Square Error of Approximation de Steiger, 1990), une valeur inférieure de 0,05 ou moins indique un bon ajustement au modèle (Hu et Bentler, 1999, cité dans Tabachnick et Fidell, 2007). Les valeurs plus élevées que 0,08 indiquent un mauvais ajustement au modèle (Browne et Cudeck, 1993, cité dans Tabachnick et Fidell, 2007).

\section{Résultats}

Le Tableau 1 présente la moyenne, l'écart-type ainsi que les degrés d'asymétrie et d'aplatissement pour chaque échelle. Pour l'estime de soi, deux dimensions (travail, conduite/moralité) présentent une moyenne supérieure à 3 (sur 4) alors que les autres se situent entre 2,3 et 3. En ce qui trait au sentiment d'autoefficacité, les dimensions sociale et scolaire présentent une moyenne se situant entre à 3,5 et 4 (sur 5) alors que pour la dimension émotionnelle, cette valeur est de 3,23. Quant à l'intention de décrocher, la moyenne est faible, soit de 2,22/7.

Tableau 1. Statistiques descriptives pour l'estime de soi, le sentiment d'autoefficacité et l'intention de décrocher $(n=391)$

\begin{tabular}{|l|l|l|l|l|l|l|l|}
\hline \multirow{2}{*}{ Variable } & \multirow{2}{*}{ M } & \multirow{2}{*}{$\begin{array}{l}\text { Asymétrie } \\
\text { ÉT }\end{array}$} & & \multicolumn{2}{|l|}{ Aplatissement } & \\
\cline { 4 - 7 } & & & Stat & ES & Stat & ES \\
\hline
\end{tabular}




\begin{tabular}{|c|c|c|c|c|c|c|}
\hline Estime de soi* & & & & & & \\
\hline Scolaire & 2,92 & 0,68 & $\mid-0,36$ & 0,12 & $-0,47$ & 0,24 \\
\hline Athlétique & 2,37 & 0,83 & 0,12 & 0,12 & $-0,94$ & 0,24 \\
\hline Travail & 3,12 & 0,68 & $-0,61$ & 0,12 & $-0,17$ & 0,24 \\
\hline Apparence physique & 2,52 & 0,85 & $-0,06$ & 0,12 & -0.87 & 0,24 \\
\hline Acceptation sociale & 2,62 & 0,70 & $-0,16$ & 0,12 & 0,51 & 0,24 \\
\hline Conduite/moralité & 3,23 & 0,63 & $-0,74$ & 0,12 & $-0,03$ & 0,25 \\
\hline Amitiés profondes & 2,93 & 0,81 & $-0,61$ & 0,12 & $-0,51$ & 0,25 \\
\hline Relations sentimentales & 2,86 & 0,68 & $-0,20$ & 0,12 & $-0,70$ & 0,24 \\
\hline Globale & 2,93 & 0,73 & $-0,48$ & 0,12 & $-0,37$ & 0,25 \\
\hline Sentiment d'autoefficaci & & & & & & \\
\hline Scolaire & 3,62 & 0,70 & $-0,53$ & 0,11 & 0,19 & 0,22 \\
\hline Émotionnelle & 3,23 & 0,76 & $-0,05$ & 0,11 & $-0,30$ & 0,22 \\
\hline Sociale & 3,84 & 0,62 & $-0,42$ & 0,11 & 0,16 & 0,22 \\
\hline Intention décrocher*** & 2,22 & 1,43 & 1,10 & 0,11 & 0,31 & 0,22 \\
\hline
\end{tabular}

Les corrélations entre les différentes dimensions des trois questionnaires sont présentées au Tableau 2. Pour l'estime de soi, trois dimensions (scolaire, conduite/ moralité et globale) présentent les corrélations les plus élevées (égale ou supérieure à $-0,35)$ avec l'intention de décrocher, la plus forte étant celle de l'estime de soi globale. Pour le sentiment d'autoefficactié, la dimension scolaire présente la corrélation la plus élevée avec l'intention de décrocher $(-0,55)$. Les corrélations les plus élevées entre l'estime de soi et le sentiment d'autoefficacité sont: scolaire vs scolaire $(0,63)$; globale vs émotionnelle $(0,58)$. Pour l'échelle d'autoefficacité sociale, trois corrélations sont plus élevées que 0,30 .

Tableau 2. Corrélations entre l'estime de soi, le sentiment d'autoefficacité et l'intention de décrocher $(n=391)$

\begin{tabular}{|l|l|l|l|l|l|l|l|l|l|l|l|l|}
\hline Variable & 2 & 3 & 4 & 5 & 6 & 7 & 8 & 9 & 10 & 11 & 12 & 13 \\
\hline Estime de soi & & & & & & & & & & & & \\
\hline 1. Scolaire & 0,05 & $0,23^{* *}$ & $0,22^{* *}$ & $0,17^{* *}$ & $0,42^{* *}$ & $0,17^{* *}$ & $0,24^{* *}$ & $0,51^{* *}$ & $0,33^{* *}$ & $0,29^{* *}$ & $0,63^{* *}$ & $-0,35^{* *}$ \\
\hline
\end{tabular}




\begin{tabular}{|c|c|c|c|c|c|c|c|c|c|c|c|}
\hline 2. Athlétique & $0,19^{* *}$ & $0,31^{* *}$ & $0,29^{* *}$ & $-0,03$ & 0,09 & $0,21^{* *}$ & $0,19^{* *}$ & $0,23^{* *}$ & $0,21^{* *}$ & 0,06 & $-0,04$ \\
\hline 3. Travail & & $0,21^{* *}$ & $0,24^{* *}$ & $0,27^{* *}$ & $0,17^{* *}$ & $0,39^{* *}$ & $0,39^{* *}$ & $0,31^{* *}$ & $0,25^{* *}$ & $0,20^{* *}$ & $-0,19^{* *}$ \\
\hline $\begin{array}{l}\text { 4. Apparence } \\
\text { physique }\end{array}$ & & & $0,22^{* *}$ & $0,20^{* *}$ & 0,09 & $0,37^{* *}$ & $0,56^{* *}$ & $0,37^{* *}$ & $0,14^{* *}$ & $0,17^{* *}$ & $-0,16^{* *}$ \\
\hline $\begin{array}{l}\text { 5. Acceptation } \\
\text { sociale }\end{array}$ & & & & 0,09 & $0,43^{* *}$ & $0,38^{* *}$ & $0,38^{* *}$ & $0,25^{* *}$ & $0,57^{* *}$ & $0,15^{* *}$ & $-0,19^{* *}$ \\
\hline $\begin{array}{l}\text { 6. Conduite/ } \\
\text { moralité }\end{array}$ & & & & & $0,17^{* *}$ & $0,25^{* *}$ & $0,51^{* *}$ & $0,27^{* *}$ & 0,09 & $0,40^{* *}$ & $-0,35^{* *}$ \\
\hline $\begin{array}{l}\text { 7. Amitiés } \\
\text { profondes }\end{array}$ & & & & & & $0,31^{* *}$ & $0,29^{* *}$ & $0,15^{* *}$ & $0,35^{* *}$ & $0,18^{* *}$ & $-0,21^{* *}$ \\
\hline $\begin{array}{l}\text { 8. Relations } \\
\text { sentimentales }\end{array}$ & & & & & & & $0,44^{* *}$ & $0,26^{* *}$ & $0,31^{* *}$ & $0,13^{* *}$ & $-0,18^{* *}$ \\
\hline 9. Globale & & & & & & & & $0,58^{* *}$ & $0,39^{* *}$ & $0,48^{* *}$ & $-0,41^{* *}$ \\
\hline $\begin{array}{l}\text { Sentiment } \\
\text { d'autoefficacité }\end{array}$ & & & & & & & & & & & \\
\hline $\begin{array}{l}10 . \\
\text { Émotionnelle }\end{array}$ & & & & & & & & & $0,42^{* *}$ & $0,32^{* *}$ & $-0,26^{* *}$ \\
\hline 11. Sociale & & & & & & & & & & $0,32^{* *}$ & $-0,29^{* *}$ \\
\hline 12. Scolaire & & & & & & & & & & & $-0,55^{* *}$ \\
\hline $\begin{array}{l}\text { 13. Intention } \\
\text { de décrocher }\end{array}$ & & & & & & & & & & & \\
\hline
\end{tabular}

L'ajustement du modèle, à neuf dimensions corrélées, aux données pour l'estime de soi présente des statistiques d'ajustement appréciables : CFI $(0,945)$, du NNFI $(0,940)$ et du RMSEA $(0,051)$. En ce qui concerne l'ajustement du modèle, à trois dimensions corrélées, aux données pour l'autoefficacité, des statistiques d'ajustement adéquates sont obtenues : CFI $(0,920)$, du NNFI $(0,910)$ et du RMSEA $(0,072)$. Enfin, l'ajustement du modèle unidimensionnel pour l'intention aux données n'a pas pu être calculé, car le nombre de degrés de liberté égale 0 .

La Figure 1 présente le modèle initial incluant les liens entre les trois concepts à l'étude (estime de soi, sentiment d'autoefficacité et intention de décrocher). Les neuf dimensions de l'estime de soi sont corrélées entre elles. Ce modèle présente des statistiques d'ajustement appréciable aux données: CFI $(0,992)$, du NNFI $(0,932)$ et du RMSEA $(0,057)$. Aucun ajout au modèle n'est proposé par le LMTEST. Aucun lien direct partant de l'estime de soi ne permet de prédire l'intention de décrocher. 
Figure 1. Modèle initial des liens entre l'estime de soi, le sentiment d'autoefficacité et l'intention de décrocher

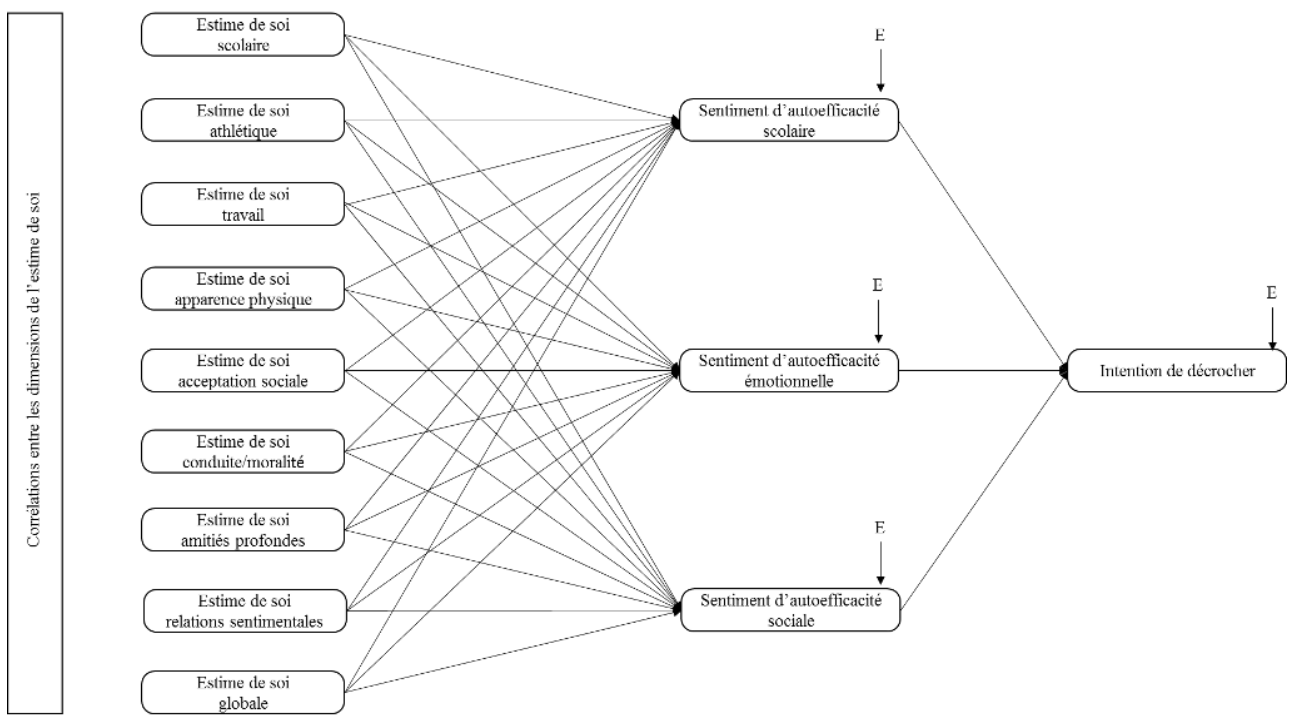

Le WTEST propose de retirer plusieurs liens et corrélations. Chacun des liens ou corrélations est retiré un à un à partir des résultats du WTEST. Le modèle final respecte le critère d'un ajustement appréciable du modèle aux données : CFI $(0,987)$, NNFI $(0,970)$ et RMSEA $(0,038)$. Ce modèle est présenté à la Figure 2 et les corrélations entre les dimensions de l'estime de soi au Tableau 3.

Figure 2. Modèle final des liens entre l'estime de soi, le sentiment d'autoefficacité et l'intention de décrocher $\left({ }^{\star} \mathrm{p}<0,05\right.$.)

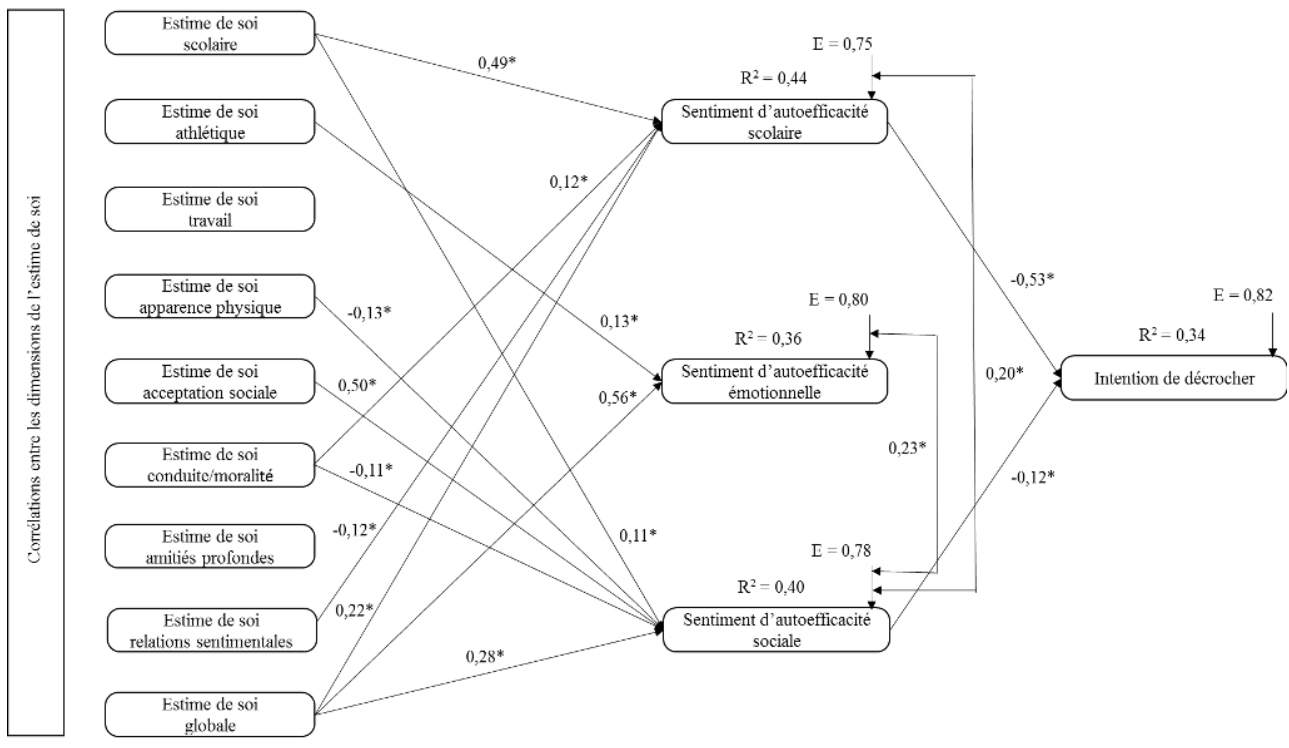

Parmi les trois dimensions du sentiment d'autoefficacité, celle scolaire permet la meilleure prédiction $(-0,53)$ de l'intention de décrocher. La dimension sociale permet de prédire légèrement $(-0,12)$ et la dimension émotionnelle n'est pas significative dans la prédiction de l'intention de décrocher. Seule l'estime de soi globale permet de prédire les trois dimensions du sentiment d'autoeffficacité; le lien pour la dimension émotionnelle étant le plus élevé $(0,56)$. L'estime de soi scolaire permet de prédire deux dimensions du sentiment d'autoefficacité : lien élevé pour scolaire $(0,49)$, mais faible 
pour sociale $(0,11)$. La dimension sociale du sentiment d'autoefficacité est prédite par cinq dimensions de l'estime de soi, le lien le plus élevé concerne la conduite/moralité $(0,50)$. Deux dimensions de l'estime de soi (amitiés profondes et travail) ne présentent aucun lien significatif avec les dimensions du sentiment d'autoefficacité.

Tableau 3. Corrélations entre les dimensions de l'estime de soi dans le modèle acheminatoire

\begin{tabular}{|c|c|c|c|c|c|c|c|c|}
\hline Variable & 2 & 3 & 4 & 5 & 6 & 7 & 8 & 9 \\
\hline \multicolumn{9}{|l|}{ Estime de soi } \\
\hline 1. Scolaire & n.s. ${ }^{* *}$ & $0,22^{*}$ & $0,22^{*}$ & $0,11^{*}$ & $0,40^{*}$ & $0,14^{*}$ & $0,19^{*}$ & $0,50^{*}$ \\
\hline 2. Athlétique & & $0,17^{*}$ & $0,31^{*}$ & $0,26^{*}$ & n.s. ${ }^{* *}$ & n.s. ${ }^{* *}$ & $0,20^{*}$ & $0,18^{*}$ \\
\hline 3. Travail & & & $0,21^{*}$ & $0,19^{*}$ & $0,26^{*}$ & $0,13^{*}$ & $0,37^{*}$ & $0,38^{*}$ \\
\hline 4. Apparence physique & & & & $0,20^{*}$ & $0,19^{*}$ & n.s.** & $0,36^{*}$ & $0,54^{*}$ \\
\hline 5. Acceptation sociale & & & & & n.s. ${ }^{* *}$ & $0,39^{*}$ & $0,34^{*}$ & $0,32^{*}$ \\
\hline 6. Conduite/moralité & & & & & & $0,12^{*}$ & $0,23^{*}$ & $0,49^{*}$ \\
\hline 7. Amitiés profondes & & & & & & & $0,27^{*}$ & $0,24^{*}$ \\
\hline 8. Relations sentimentales & & & & & & & & $0,42^{*}$ \\
\hline 9. Globale & & & & & & & & \\
\hline \multicolumn{9}{|l|}{$*_{p}<0,05}$. \\
\hline
\end{tabular}

Bref, l'analyse acheminatoire du modèle proposé présente des statistiques d'ajustement adéquates aux données. Elle révèle des liens indirects entre l'estime de soi et l'intention de décrocher d'étudiants du collégial de même que le rôle d'intermédiaire du sentiment d'autoefficacité dans ces relations.

\section{Discussion}

La présente étude a permis de bonifier la compréhension des causes possibles du décrochage scolaire. Plus précisément, elle a vérifié un modèle théorique exploratoire expliquant les relations entre l'estime de soi et l'intention de décrocher d'étudiants du collégial, tout en examinant le rôle que joue leur sentiment d'autoefficacité dans ces relations.

\subsection{Relations entre l'estime de soi et l'intention de décrocher}

Les résultats indiquent des relations entre l'estime de soi et l'intention de décrocher d'étudiants du collégial. Plus précisément, ils montrent des liens négatifs pour huit des 
neuf dimensions de l'estime de soi et l'intention de décrocher. En effet, les résultats qui se rapportent à l'estime de soi relative aux domaines scolaire, relié au travail, relié à l'apparence physique, relié à l'acceptation sociale, relié aux amitiés profondes, relié aux relations sentimentales, relié à la conduite/moralité ainsi qu'à l'estime de soi globale sont corrélées de façon négative et statistiquement significative à l'intention de décrocher. L'estime de soi relative au domaine athlétique, quant à elle, présente une relation non statistiquement significative avec l'intention de décrocher.

Ces résultats sont cohérents avec ceux des études antérieures. En effet, ces dernières indiquent que l'estime de soi des étudiants est reliée à différentes variables relatives au milieu scolaire (Bardou et al., 2012; Kandemir et al., 2014; Murray et Kennedy-Lightsey, 2013; Perterson-Graziose et al, 2013). De même, les résultats appuient l'analyse des éléments théoriques qui suggère que l'estime de soi des étudiants du collégial puisse être reliée à leur intention de décrocher. En effet, tel que mentionné précédemment, l'estime de soi globale serait fortement liée aux affects de l'adolescent (Harter, 1999) et des affects négatifs augmenteraient le risque de décrochage scolaire (Janosz, 2000).

Toutefois, il faut rappeler que plusieurs autres facteurs peuvent contribuer simultanément à l'intention de décrocher. L'estime de soi des étudiants du collégial ne serait donc pas nécessairement le seul antécédent de cette intention de décrocher. À cet égard, la faible intensité des relations observées peut constituer une indication dans ce sens. Bref, les résultats indiquent tout de même que l'intention de décrocher d'étudiants du collégial puisse être tributaire, du moins en partie, de leur estime de soi.

\subsection{Rôle intermédiaire du sentiment d'autoefficacité}

Les résultats montrent des relations entre l'estime de soi et l'intention de décrocher, entre l'estime de soi et le sentiment d'autoefficacité ainsi qu'entre le sentiment d'autoefficacité et l'intention de décrocher d'étudiants du collégial. Plus précisément, ils montrent que le sentiment d'autoefficacité joue un rôle intermédiaire dans les relations entre leur estime de soi et leur intention de décrocher. À cet égard, les analyses acheminatoires permettent de préciser ces résultats en démontrant que les relations entre l'estime de soi et l'intention de décrocher sont indirectes et que le sentiment d'autoefficacité joue un rôle d'intermédiaire dans ces relations.

Ces résultats sont en accord avec ceux des recherches recensées. En effet, certaines présentent des relations entre une des dimensions (scolaire, émotionnelle ou sociale) du sentiment d'autoefficacité et des variables reliées au domaine scolaire (Adeyinka et al., 2011; Alivernini et Lucidi, 2011; Faurie, 2012; Hsieh et al., 2007; Kandemir et al., 2014; Ladd, 1990; Qualter et al., 2012). Ces résultats sont également cohérents avec ceux d'autres travaux antérieurs (Kandemir et al., 2014; Khan et al., 2015; McKay et al., 2014; Peterson-Graziose et al., 2013) qui mettent en lumière des liens entre l'estime de soi et le sentiment d'autoefficacité. Ils concordent aussi avec les résultats de l'étude de McKay et al. (2014) qui montrent que l'estime de soi prédit le sentiment d'autoefficacité, contrairement à ceux de Hajloo (2014) qui suggèrent le contraire.

Les résultats appuient les propos de McKay et al. (2014) à l'effet que l'estime de soi aurait un impact sur le sentiment d'autoefficacité. Les résultats soutiennent également l'analyse de la théorie du sentiment d'autoefficacité de Bandura (2007). Cette analyse suggère que, selon la force de son sentiment d'autoefficacité, un étudiant du collégial aurait tendance à faire le choix de poursuivre ou d'abandonner ses études. De même, le 
niveau du sentiment d'autoefficacité affecterait sa motivation à poursuivre ou à abandonner ses études.

41 En somme, bien que l'estime de soi et le sentiment d'autoefficacité soient susceptibles, individuellement, d'influer sur l'intention de décrocher, leur combinaison semble avoir davantage d'influence sur cette intention. De même, selon le modèle final, l'influence du sentiment d'autoefficacité sur l'intention de décrocher est directe alors que celle de l'estime de soi est indirecte. Les résultats suggèrent donc que plus l'estime de soi et le sentiment d'efficacité des étudiants du collégial sont forts, moins ces derniers auront l'intention de décrocher et vice et versa.

\section{Conclusion}

Bien que la société québécoise soit mobilisée autour du décrochage scolaire au secondaire depuis plusieurs années, il n'en est pas de même pour le collégial (Michel Perron, cité dans Dion-Viens, 2017). En effet, cet enjeu semble plutôt peu considéré et c'est pourquoi des pistes d'intervention et de recherche sur le décrochage scolaire au collégial demeurent de première importance.

qui a trait aux interventions, les résultats obtenus dans la présente étude constituent une piste intéressante, notamment pour la formation (initiale ou continue) offerte par les universités aux enseignants du collégial. En effet, comme une estime de soi élevée chez les jeunes favoriserait la persévérance scolaire (Bardou et al., 2012; Perterson-Graziose et al., 2013), une partie de cette formation doit s'attarder aux comportements et attitudes que les enseignants doivent adopter afin de favoriser le développement de cette estime chez leurs étudiants. Comme le mentionne Harter (1999), l'évaluation que font les jeunes de leurs compétences dans les domaines les plus importants pour eux ainsi que le soutien qu'ils perçoivent des personnes significatives de leur vie constituent des déterminants importants de leur estime de soi. Les formateurs universitaires doivent alors encourager les enseignants du collégial à repérer, à valoriser et à renforcer les intérêts et les habiletés de leurs étudiants. Aussi, les enseignants doivent faire preuve d'ouverture, d'écoute et de compréhension afin que leurs étudiants se sentent soutenus dans leur cheminement scolaire. Une telle formation contribuerait certainement à améliorer l'approche maître-élève des enseignants et, par le fait même, à encourager les étudiants dans la poursuite de leurs études.

De plus, comme les résultats de l'étude le montrent, des dimensions du sentiment d'autoefficacité joue un rôle médiateur dans les relations entre certaines dimensions de l'estime de soi et l'intention de décrocher, il apparaît important de trouver des moyens afin d'améliorer ce sentiment chez les étudiants du collégial. Pour ce faire, il semble impératif de sensibiliser les enseignants à cette situation et les former quant aux différentes sources possibles du sentiment d'autoefficacité proposées par Bandura (2007). En raison de cette sensibilisation et formation, ils seront en mesure d'utiliser des stratégies afin de favoriser le développement du sentiment d'autoefficacité de leurs étudiants. Comme le mentionne Bandura (2007), les apprenants qui présentent un fort sentiment d'autoefficacité seraient plus enclins à persévérer, à être motiver, à performer intellectuellement et à atteindre des objectifs élevés que ceux dont ce sentiment est faible. 

domaine du décrochage scolaire au collégial, elle comporte tout de même certaines limites. D'abord, étant donné le manque d'études sur le sujet et la nature même des variables, qui d'un point de vue éthique peuvent difficilement être manipulées, la méthode corrélationnelle est celle privilégiée. Les résultats de ce type de recherche ne peuvent mener à une conclusion de cause à effet; l'interprétation de ses résultats se limitant à indiquer le degré et la nature des relations entre les variables (Fortin, 2010). De plus, comme le plan de l'expérience est à mesure unique, il n'est pas possible de déterminer si les relations observées se maintiennent dans le temps. Ensuite, un échantillon non probabiliste par choix raisonné ou intentionnel tel qu'utilisé ne permet pas de préciser l'erreur d'échantillonnage. Cet échantillon risque donc d'être moins représentatif que l'échantillon probabiliste (Beaud, 2003; Contendriopoulos et al., 1990; Fortin, 2010). De plus, le taux de réponse de la présente recherche est très faible (5,7 \%), et ce, malgré une relance par courriel. Le type d'échantillonnage et le faible taux de participation limitent la généralisation des résultats à la population (Fortin, 2010) des étudiants du collégial ainsi que le nombre de variables qu'il est possible de considérer dans les analyses par équations structurelles comme celle réalisées dans le cadre de la présente étude.

À la lumière des résultats obtenus et des limites soulevées, certaines pistes émergent pour la recherche future. En effet, il semble opportun de refaire cette étude auprès de plusieurs échantillons d'étudiants afin de favoriser la généralisation des résultats à la population des étudiants du collégial. De plus, afin de vérifier si les relations entre les trois variables persistent dans le temps, il serait intéressant que le plan de l'expérience prévoie des mesures répétées. Enfin, étant donné les répercussions potentielles du décrochage scolaire au collégial et le manque de littérature sur ses causes, il serait primordial que d'autres études empiriques soient menées auprès de grands échantillons afin de relever d'autres facteurs explicatifs possibles, et ce, en fonction du genre et de l'âge, par exemple. Les résultats de la présente étude suggèrent que le sentiment d'autoefficacité soit en relation plus étroite avec l'intention de décrocher qu'un autre facteur personnel, c'est-à-dire l'estime de soi. Il serait donc pertinent d'examiner les relations qui existent entre l'intention de décrocher et d'autres facteurs personnels relatifs à l'étudiant.

\section{BIBLIOGRAPHY}

Adeyinka, T. Adedeji, T. et Sam Olufemi, A. (2011). Locus of control, interest in schooling and selfefficacy as predictors of academic achievement among junior secondary school students in Osun State, Nigeria. New Horizons in Education, 59(1), 25-37.

Aliverni, F. et Lucidi, F. (2011). Relationship between social context, self-efficacy, motivation, academic achievement, and intention to drop out of high school : a longitudinal study. The Journal of Educational Research, 104(4), 241-252.

Bandura, A. (1997). Self-efficacy: The exercise of control. W. H. Freeman and Company. 
Bandura, A. (2007). Autoefficacité : le sentiment d'efficacité personnelle. De Boeck.

Bardou, E., Oubrayrie-Roussel, N. et Lescarret, O. (2012). Estime de soi et démobilisation scolaire des adolescents. Neuropsychiatrie de l'enfance et de l'adolescence, 60(6), 435-440.

Beaud, J.-P. (2003). L'échantillonnage. Dans B. Gauthier (dir.), Recherche sociale : de la problématique à la collecte des données, (p. 211-242). Presses de l'Université du Québec.

Bentler, P. M. (1990). Comparative fit indexes in structural models. Psychological Bulletin, 107(2), 238-246.

Bentler, P. M. et Wu, E. J. C. (2006). EQS for Windows: User's guide. Multivariate Software, Inc. Bouffard-Bouchard, T. (1992). Relation entre la savoir stratégique, l'évaluation de soi et le sentiment d'autoefficacité, et leur influence dans une tâche de lecture. Enfance, 45(1-2), 63-78.

Bouffard, T., Seidah, A., Mcintyre, M., Boivin, M., Vezeau, C. et Cantin, S. (2002). Mesure de l'estime de soi à l'adolescence : version canadienne-française du Self-Perception Profile for Adolescents de Harter. Revue canadienne des sciences du comportement, 34(3), 158-162.

Browne, M. W. et Cudeck, R. (1993). Alternative ways of assessing model fit. Dans K. A. Bollen et J. S. Long (dir.), Testing Structural Models (p. 136-162). Sage Publications.

Byrne, B. M. (2006). Structural Equation Modeling with EQS: Basic Concepts, Applications, and Programming ( $2^{\mathrm{e}}$ éd.). Lawrence Erlbaum Associates, Publishers.

Capron, I. P. (2012). Le sentiment d'efficacité personnelle et l'apprentissage des langues. Les Cahiers de l'Acedle, 9(1), 75-92.

Choi, N. (2003). Further examination of the self-efficacy scale. Psychological Reports, 92(2), 473-480.

Chou, C. P. et Bentler, P. M. (1990). Model modification in covariance structure modeling: A comparison among likelihood ratio, Lagrange multiplier, and Wald tests. Multivariate Behavioral Research, 25(1), 115-136.

Coleman, P. K. (2003). Perceptions of parent-child attachment, social self-efficacy, and peer relationships in middle childhood. Infant and Child Development, 12(4), 351-368.

Comité Mauriciens sur la Persévérance et la Réussite Scolaire (COMPERES). (2013). Les effets du décrochage. Trois-Rivières, QC : COMPERES.

Comité Régional pour la Valorisation de l'Éducation (CRÉVALE). (2013). Conséquences $d u$ décrochage. http://www.crevale.org/index.jsp?p=102

Contendriopoulos, A.-P., Champagne, F., Potvin, L., Denis, J.-L. et Boyle, P. (1990). Savoir préparer une recherche : la définir, la structurer, la financer. Les Presses de l'Université de Montréal.

Dion-Viens, D. (2017, 2 juin). Le décrochage en hausse au cégep. Le Journal de Québec. https:// www.journaldequebec.com/2017/06/02/le-decrochage-en-hausse-au-cegep

Dumont, M., Leclerc, D. et Deslandes, R. (2003). Ressources personnelles et détresse psychologique en lien avec le rendement scolaire et le stress chez des élèves de quatrième secondaire. Revue canadienne des sciences du comportement, 35(4), 254-267.

Faurie, I. (2012). Sentiments d'efficacité personnelle et dynamique du projet professionnel. Psychologie du travail et des organisations, 17(1), 37-60.

Fortin, M. -F. (2010). Fondements et étapes du processus de recherche. Méthodes quantitatives et qualitatives ( 2 éd.). Chenelière Éducation. 
Fortin, L., Royer, E., Potvin, P., Marcotte, D. et Yergeau, E. (2004). La prédiction du risque de décrochage scolaire au secondaire : facteurs personnels, familiaux et scolaires. Canadian Journal of Behavioral Science, 36(3), 219-231.

Galand, B. et Vanlede, M. (2004). Le sentiment d'autoefficacité personnelle dans l'apprentissage et la formation : Quel rôle joue-t-il? D'où vient-il? Comment intervenir? Savoirs, 5, hors-série), 91-116.

Gaudreault, M., Labrosse, J., Tessier, S., Gaudreault, M. et Arbour, N. (2014). L'intégration aux études et l'engagement scolaire des collégiens : enquête menée dans les régions de Lanaudière, de la Mauricie et du Saguenay-Lac-Saint-Jean. ÉCOBES - Recherche et transfert. Cégep de Jonquière, Saguenay, QC, Canada.

Gurtner, J.-L., Gorga, A., Monnard, I. et Ntamakilirio, L. (2001). Évolution de diverses composantes de la motivation pour le travail scolaire au cours de l'adolescence : brève synthèse à l'intention des autorités scolaires et des enseignants. Université de Fribourg.

Hajloo, N. (2014). Relationships between self-efficacy, self-esteem and procrastination in undergraduate psychology students. Iran Journal of Psychiatry Behavioral Sciences, 8(3), 42-49.

Hardre, P. L. et Reeve, J. (2003). A motivational model of rural students' intentions to persist in, versus drop out of high school. Journal of Educational Psychology, 95(2), 347-356.

Harter, S. (1988). Self-perception profile for adolescents [document inédit]. University of Denver. Harter, S. (1999). The Construction of the Self. A Developmental Perspective. The Guilford Press. Hsieh, P., Sullivan, J. R. et Guerra, N. S. (2007). A closer look at college students : self-efficacy and goal orientation. Journal of Advanced Academics, 18(3), 454-476.

Hu, L. T. et Bentler, P. M. (1999). Cutoff criteria for fit indexes in covariance structure analysis: Conventional criteria versus new alternatives. Structural Equation Modeling : A Multidisciplinary Journal, 6, 1-55.

Janosz, M. (2000). L'abandon scolaire chez les adolescents: perspective nord-américaine. VEI Enjeux, 122(9), 105-127.

Janosz, M., Le Blanc, M., Boulerice, B. et Tremblay, R. E. (1997). Disentangling the weight of school dropout predictors : A test on two longitudinal samples. Journal of Youth and Adolescence, 26(6), 733-762.

Joet, G. (2010). Le sentiment d'autoefficacité en primaire : de son élaboration à son impact sur la scolarité des élèves [thèse de doctorat inédite]. Université Pierre-Mendes-France (Grenoble II), Genoble, France.

Kandermir, M., Ilhan, T., Ozpolat, A. R. et Palanci, M. (2014). Analysis of academic self-efficacy, self-esteem and coping with stress skills predictive power on academic procrastination. Academic Journal, 9(5), 146-152.

Khan, A., Fleva, E. et Qazi, T. (2015) Role of self-esteem and general self-efficacy in teacher's efficacy in primary schools. Psychology, 6(1), 117-125.

Kirk, B. A., Schutte, N. S. et Hine, D. W. (2008). Development and preliminary validation of an emotional self-efficacy scale. Personality and Individual Differences, 45(5), 432-436.

Ladd, G. W. (1990). Having friends, keeping friends, making friends, and being liked by peers in the classroom : Predictors of children's early school adjustment? Child Development, 61, 1081-1100. 
Lecompte, J. (2004). Les applications du sentiment d'efficacité personnelle. Savoirs: Revue Internationale de Recherche en Éducation et Formation des Adultes, Hors-série : Autour de l'œuvre d'Albert Bandura, 59-90.

Lee, S. Y., Poon, W. Y. et Bentler, P. M. (1995). A two-stage estimation of structural equation models with continuous and polytomous variables. British Journal of Mathematical and Statistical Psychology, 48(2), 339-358.

Legendre, R. (2005). Dictionnaire actuel de l'éducation ( $3^{\mathrm{e}}$ éd.). Guérin Éditeur.

Little, T. D., Rhemtulla, M., Gibson, K. et Schoemann, A. M. (2013). Why items versus parcels controversy needn't be one? Psychological Reports, 18(3), 285-300.

Luszczynska, A., Gutiérrez-Dona, B. et Schwarzer, R. (2005). General self-efficacy in various domains of human functioning : Evidence from five countries. International Journal of Psychology, 40(2), 80-89.

Marsh, H. W., Hau, K. T. et Balla, J. (1997, mars). Is more ever too much : The number of indicators per factor in confirmatory factor analysis [communication]. Rencontre annuelle du American Educational Research Association (AERA), Chicago, IL, États-Unis.

McKay, M. T., Dempster, M. et Byrne, D. G. (2014). An examination of the relationship between self-efficacy and stress in adolescents : the role of gender and self-esteem. Journal of Youth Studies, 17(9), 1131-1151.

Ménard, L. J. (2009). Au-delà des chiffres : une affaire de cœur. L'épaule à la roue : comment le Québec peut se mobiliser contre le décrochage scolaire. BMO Groupe financier.

Ministère de l'Éducation du Loisir et du Sport (MELS). (2010). Les indicateurs de l'éducation. http:// www.mels.gouv.qc.ca/sections/publications/publications/SICA/DRSI/ IndicateursEducation2010_f.pdf

Muchielli, A. (2006). Les motivations. Presses universitaires de France (PUF).

Muris, P. (2001). A brief questionnaire for measuring self-efficacy in youths. Journal of Psychopathology and Behavioral Assessment, 23(3), 145-149.

Murray, C. L. et Kennedy-Lightsey, C. D. (2013). Should I stay or go? Student identity gaps, feelings, and intent to leave. Communication Research Reports, 30(2), 96-105.

Peterson-Graziose, V., Bryer, J. et Nikolaidou, M. (2013). Self-esteem and self-efficacy as predictors of attrition in associate degree nursing students. Journal of Nursing Education, 52(6), 351-354.

Projet Partenaires pour la réussite éducative en Estrie (PRÉE). (2012). Quelques définitions pour s'y retrouver. http://www.reussiteeducativeestrie.ca

Qualter, P., Gardner, K., Pope, D., Hutchinson, J. M. et Whiteley, H. E. (2012). Ability emotional intelligence, trait emotional intelligence, and academic success in British secondary schools: A 5year longitudinal study. Learning and Individual Differences, 22(1), 83-91.

Rapport à l'école au collégial. (2021). Des pistes pour persévérer dans l'égalité. Gaspésie et îles-de-laMadeleine : Persévérer dans l'égalité. https://enseignerlegalite.com/

Robertson, A. et Collerette, P. (2005). L'abandon scolaire au secondaire : prévention et interventions. Revue des sciences de l'éducation, 31(3), 687-707. 
Roy, J., Bouchard. J. et Turcotte, M.-A. (2010). Filles et garçons au collégial : des univers parallèles. Masculinités et Société et Observatoire Jeunes et Société., Cégep de Sainte-Foy Québec, QC, Canada

Schumacker, R. E. et Lomax, R. G. (1996). A beginner's guide to structural equation modeling. Lawrence Erlbaum Associates.

Shaienks, D., Gluszynski, T. et Bayard, J. (2008). Les études postsecondaires - participation et décrochage : différences entre l'université, le collège et les autres types d'établissements postsecondaires. Statistique canada. https://www150.statcan.gc.ca/n1/pub/81-595-m/81-595-m2008070-fra.htm Shi, Q. et Steen, S. (2012) Using the Achieving Success Everyday (ASE) group model to promote self-esteem and acadamic achivement for English as a Second Language (ESL) students. Professional School Counseling, 13(3), 63-70.

Steiger, J. H. (1990). Structural model evaluation and modification: an interval estimation approach. Multivariate Behavioural Research, 25(2), 173-180.

Suldo, S. M. et Shaffer, E. J. (2007). Evaluation of the self-efficacy questionnaire for children in two samples of American adolescents. Journal of Psychoeducational Assessment, 25(4), 341-355.

Tabachnick, B. G. et Fidell, L. S. (2007). Using Multivariate Statistics. Allyn and Bacon/Pearson Education.

Tucker, L. R. et Lewis, C. (1973). A reliability coefficient for maximum likelihood factor analysis. Psychometrika, 38, 1-10.

\section{NOTES}

1. Compte-tenu du peu de documents disponibles sur le décrochage scolaire au collégial, des écrits traitant du décrochage scolaire au secondaire ont été intégrés aux parties 2.2 et 2.3 .

\section{ABSTRACTS}

For several years, the province of Quebec has been mobilizing to fight school dropouts. Because of the negative repercussions generates both at the individual and societal levels this growing phenomenon remains a major issue. The purpose of this study is to highlight the personal factors underlying school dropouts. More specifically, its purpose is to explore the relationships between self-esteem and intent to drop out among college students, as well as the role of self-efficacy on these relationships. Three hundred and ninety-one (391) students from three colleges (two public and one private) participated in the research. The French Canadian version of Bouffard et al. (2002) of Self-Perception Profile for Adolescents (SPPA, Harter, 1988), the teenager's adaptation by Suldo and Shaffer (2007) of the Self-Efficacy Questionnaire for Children (SEQ-C, Muris, 2001) and the translation of the three items proposed by Hardre and Reeve (2003) allow to measure self-esteem, self-efficacy, and intent to drop out, respectively. The results suggest that college students' self-esteem and sense of efficacy are negatively related to intent to drop out. More specifically, the path analysis of the model presents adequate adjustment statistics of the model 
to the datas. It reveals indirect links between self-esteem and intent to drop out as well as the intermediary role of self-efficacy in these relationships., These results are discussed in light of the underlying theories as well as from previous studies.

Depuis plusieurs années, la province de Québec se mobilise afin de lutter contre le décrochage scolaire. Par les nombreuses répercussions négatives qu'il engendre tant sur le plan individuel que sociétal, ce phénomène grandissant demeure un enjeu de premier plan. Le but de la présente étude est de mettre en lumière des facteurs personnels sous-jacents au décrochage scolaire. Plus précisément, son objectif consiste à explorer les relations entre l'estime de soi et l'intention de décrocher d'étudiants du collégial, de même que d'identifier le rôle du sentiment d'autoefficacité dans ces relations. Trois cent quatre-vingt-onze (391) étudiants, provenant de trois établissements collégiaux (deux publics et un privé), ont participé à la recherche. La version canadienne française de Bouffard et al. (2002) du Self-Perception Profil for Adolescents (SPPA, Harter, 1988), l'adaptation réalisée pour des adolescents par Suldo et Shaffer (2007) du Self-Efficacy Questionnaire for Children (SEQ-C, Muris, 2001) ainsi que la traduction des trois énoncés que proposent Hardre et Reeve (2003) permettent de mesurer, respectivement, l'estime de soi, le sentiment d'autoefficacité et l'intention de décrocher. Les résultats montrent que l'estime de soi et le sentiment d'efficacité des étudiants du collégial présentent des corrélations négatives avec l'intention de décrocher. Plus précisément, l'analyse acheminatoire présente des statistiques d'ajustement adéquates du modèle aux données. Elle révèle l'existence de liens indirects entre l'estime de soi et l'intention de décrocher, de même que le rôle d'intermédiaire du sentiment d'autoefficacité dans ces relations. Ces résultats sont discutés à la lumière des théories sousjacentes ainsi qu'à partir d'études antérieures.

\section{INDEX}

Mots-clés: collégial, décrochage scolaire, estime de soi, étudiants, intention de décrocher, sentiment d'autoefficacité

\section{AUTHORS}

\section{STÉPHANE THIBODEAU}

Université du Québec à Trois-Rivières, Trois-Rivières, Québec, Canada,

Stephane.Thibodeau@uqtr.ca

\section{ELISABETH GIGUÈRE}

Cégep de Trois-Rivières, Trois-Rivières, Québec, Canada, Elisabeth.Giguere@cegeptr.qc.ca

\section{SONIA LEFEBVRE}

Université du Québec à Trois-Rivières, Trois-Rivières, Québec, Canada, Sonia.Lefebvre@uqtr.ca

\section{ERIC FRENETTE}

Université Laval, Québec, Québec, Canada, Eric.Frenette@fse.ulaval.ca 